\title{
Quantitative differences in developmental profiles of spontaneous activity in cortical and hippocampal cultures
}

\author{
Paul Charlesworth ${ }^{1,2+}$, Ellese Cotterill ${ }^{3 \dagger}$, Andrew Morton ${ }^{1,4}$, Seth GN Grant ${ }^{5}$ and Stephen J Eglen ${ }^{3 *}$
}

\begin{abstract}
Background: Neural circuits can spontaneously generate complex spatiotemporal firing patterns during development. This spontaneous activity is thought to help guide development of the nervous system. In this study, we had two aims. First, to characterise the changes in spontaneous activity in cultures of developing networks of either hippocampal or cortical neurons dissociated from mouse. Second, to assess whether there are any functional differences in the patterns of activity in hippocampal and cortical networks.

Results: We used multielectrode arrays to record the development of spontaneous activity in cultured networks of either hippocampal or cortical neurons every 2 or 3 days for the first month after plating. Within a few days of culturing, networks exhibited spontaneous activity. This activity strengthened and then stabilised typically around 21 days in vitro. We quantified the activity patterns in hippocampal and cortical networks using 11 features. Three out of 11 features showed striking differences in activity between hippocampal and cortical networks: (1) interburst intervals are less variable in spike trains from hippocampal cultures; (2) hippocampal networks have higher correlations and (3) hippocampal networks generate more robust theta-bursting patterns. Machine-learning techniques confirmed that these differences in patterning are sufficient to classify recordings reliably at any given age as either hippocampal or cortical networks.
\end{abstract}

Conclusions: Although cultured networks of hippocampal and cortical networks both generate spontaneous activity that changes over time, at any given time we can reliably detect differences in the activity patterns. We anticipate that this quantitative framework could have applications in many areas, including neurotoxicity testing and for characterising the phenotype of different mutant mice. All code and data relating to this report are freely available for others to use.

Keywords: Multielectrode array, Spontaneous activity, Cortex, Hippocampus, Principal component analysis, Support vector machine, Classification tree

\section{Background}

During development, many parts of the nervous system generate patterns of spontaneous activity. These patterns of activity are thought to be instructive in the assembly of neural connectivity, for example by driving activity-dependent mechanisms [1]. To date, most recordings of spontaneous activity have been in vitro,

\footnotetext{
*Correspondence: sje30@cam.ac.uk

tEqual contributors

${ }^{3}$ Cambridge Computational Biology Institute, University of Cambridge,

Wilberforce Road, Cambridge, CB3 OWA, UK

Full list of author information is available at the end of the article
}

although spontaneous activity in vivo has also been reported in hippocampus and in several cortical areas [2-6]. In vitro recordings are typically made with multielectrode arrays (MEAs), which contain at least 60 electrodes. These recordings allow us to assess activity at a range of levels from the single unit to the network. Beyond their relevance for understanding how activity might guide development of the nervous system, spontaneous activity recordings have also been used as an assay for network performance in applied settings, like neurotoxicity screening [7]. 
In recent years there has been significant interest in measuring the developmental patterns of spontaneous activity in networks cultured from neurons in control and experimental conditions [7-11]. Although many properties of spontaneous activity have been reported, we do not yet have a systematic sense of how these features change across development, or which features of neural activity are useful for describing the observed patterns of activity.

To address both these questions, we have cultured two types of network on MEAs and recorded their activity every 2 to 3 days up to around 1 month post-plating of neurons onto the array. In the first type of network, we cultured hippocampal neurons taken from embryonic mice. The second type of network was created using exactly the same protocol except with neurons dissected from cortex. Recordings of spontaneous activity from both types of network were quantified using 11 different features at the level of individual electrodes, pairs of electrodes or the entire array. We found that hippocampal networks tend to generate more regular bursting activity, including theta bursts, and more correlated activity than the corresponding cortical networks at the same age.

\section{Results}

\section{Development of spontaneous activity}

Within 7 days of culturing neurons on MEAs, spontaneous activity can be reliably recorded (Figure 1) from both hippocampal and cortical networks. As development progresses, we find that the firing rate increases, and that the frequency of bursting increases. To quantify these differences, we have used a range of measures (Figure 2) to assess the activity at a single-electrode level, pairwise and at the level of the entire network. All of these measures are defined in the methods.

\section{Overall firing rates}

During development, there are slight, statistically significant differences in firing rates, with median firing rates being slightly higher for hippocampal networks, but overall there are no key differences at maturity (Figure 3A).

\section{Bursting properties}

Neurons typically fire in bursts, and are thought to be a reliable unit of neuronal information for functions such as coincidence detection and synaptic modification [12]. We find that bursts emerge around 7 DIV (days in vitro) and strengthen until about $14 \mathrm{DIV}$ after which the bursting properties tend to stabilise. Among the bursting properties that we have measured, two factors seem to differentiate hippocampal and cortical networks. First, there is a higher fraction of spikes occurring within bursts for hippocampal networks (Figure 3E), although the difference is no longer significant by 28 DIV. Second, the spike trains from hippocampal networks seem to be more regular, as indicated by the lower coefficient of variation for interburst intervals (CV of IBIs) (Figure 3F). The other burst-based measures that we calculated, namely withinburst firing rate (Figure $3 \mathrm{~B}$ ), burst rate (Figure $3 \mathrm{C}$ ) and duration (Figure 3D) show weaker differences between the two types of network.

\section{Network activity}

The previous measures analysed spiking data independently on each electrode. As a first approximation to assessing network activity, we used the concept of network spikes [13] to define the degree to which activity is coordinated across the entire array. At any time $t$ we count the number of active electrodes; when this count exceeds a threshold, we say that a network spike has occurred. We measured three properties of these network spikes: their rate (per minute), their duration and their peak amplitude. Hippocampal networks tend to have more network spikes than cortical networks (Figure 3G) and the network spikes involve more electrodes across development (Figure $3 \mathrm{H}$ ). The hippocampal network spikes tend to last
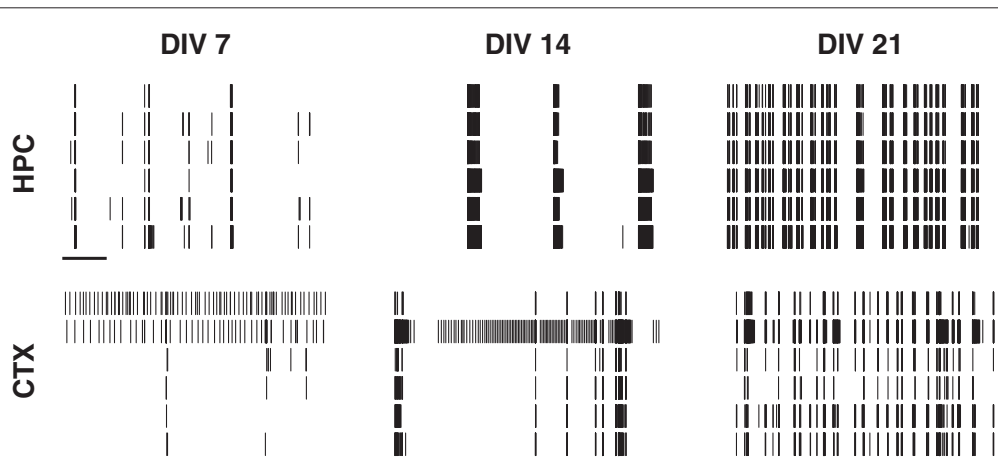

Figure 1 Examples of spontaneous activity in developing cultures. Top row: Hippocampal (HPC) cultures. Bottom row: Cortical (CTX) cultures. Each column represents one day in vitro (DIV). Within each raster plot, one row represents the spike train from one electrode; six (out of typically 59) electrodes are shown. Scale bar for all rasters is 10 s. CTX, cortex; DIV, days in vitro; HPC, hippocampus. 


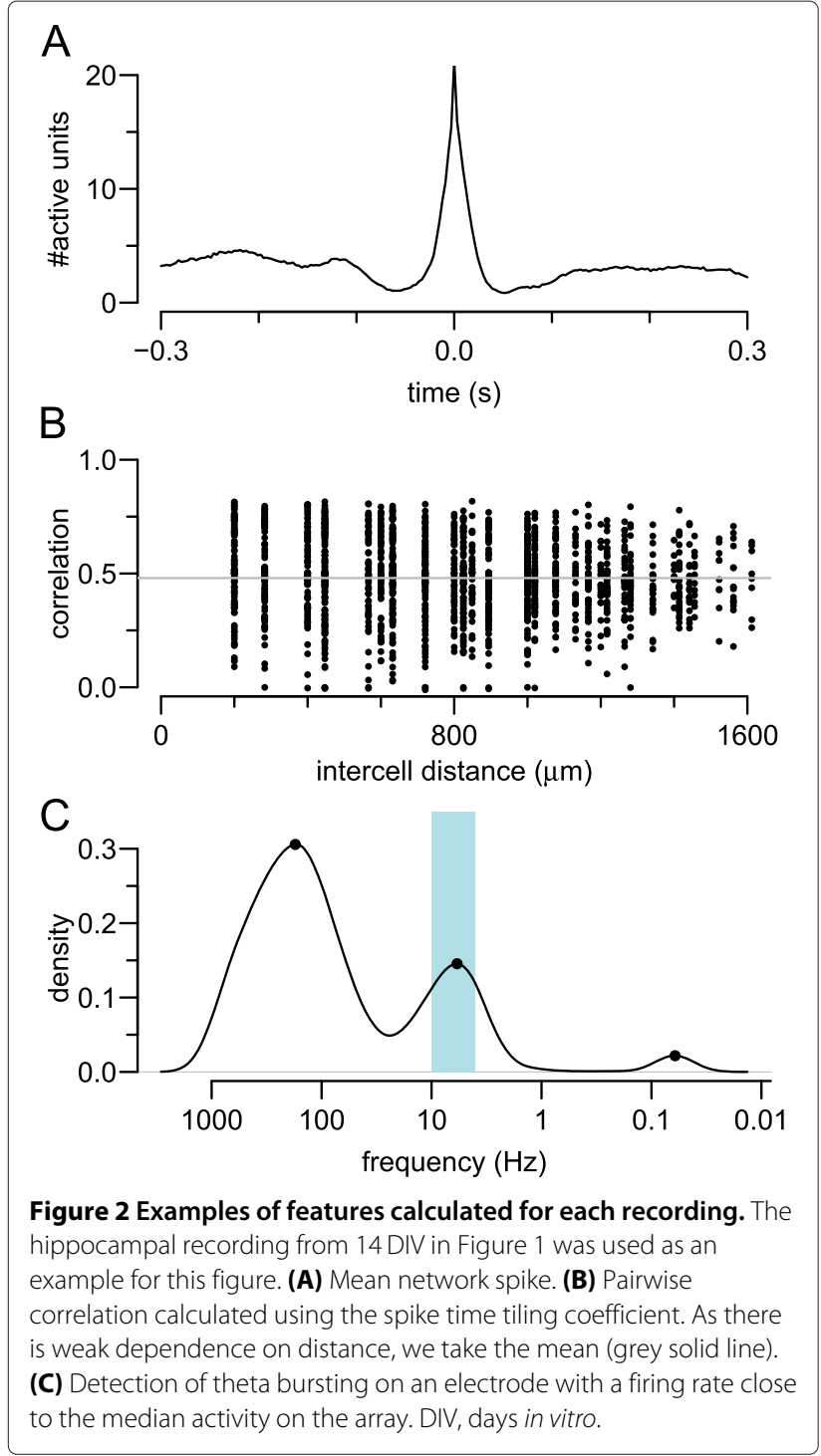

slightly longer, although this is not consistent across development (Figure 3I). Overall, this suggests that network activity tends to be stronger and more coordinated in hippocampal than cortical networks.

\section{Pairwise correlations}

As a further method to detect coincident activity on electrodes, we computed correlation coefficients for all possible pairs of electrodes on the array. For any pair of spike trains, we computed the spike time tiling coefficient, as this measure is particularly well suited for relatively sparse spike trains [14]. For $N$ (typically 59) electrodes on the array we compute $N(N-1) / 2$ correlation coefficients (i.e. ignoring autocorrelations) and plot them as a function of the distance separating the two electrodes (Figure 2B). This technique has been used in studies of spontaneous activity in developing retina, and often reveals that correlations are dependent on distance, typically following a decaying-exponential profile [15]. However, we found that there is little, if any, distance dependence upon the correlation coefficients (Figure 2B), similar to that reported before [16]. We therefore decided to compute the average of all pairwise correlations.

Across all developmental ages, we find that the mean correlation is higher in hippocampal than in cortical networks (Figure 3J). From 7 DIV to 14 DIV, we see that the mean correlation becomes reliably stronger; after $14 \mathrm{DIV}$ the correlations tend to stabilise.

\section{Presence of theta bursting}

The theta rhythm is a prominent 4 to $10 \mathrm{~Hz}$ oscillation measured in the hippocampus, and is thought to be involved in a range of neural functions [17]. We decided to examine whether our networks exhibited such oscillations by checking for peaks in the log interspike interval (ISI) histogram in the range 0.1 to $0.25 \mathrm{~s}$. Figure $2 \mathrm{~B}$ shows an example of one electrode (recording from a 14 DIV hippocampal network) that exhibited theta bursting. Our approach was to measure the fraction of electrodes exhibiting theta bursting. Perhaps the most striking feature that discriminates hippocampal from cortical networks is the presence of theta bursting in hippocampal networks. Although only about $10 \%$ of electrodes in hippocampal networks are classified as theta bursting at 7 DIV, after 11 DIV theta bursting is found on $50 \%$ to $75 \%$ of electrodes (Figure $3 \mathrm{~K}$ ). By contrast, most electrodes in cortical networks do not detect theta bursting, except at 25 DIV.

\section{Discrimination of hippocampal and cortical networks}

Each of the 11 features documented in Figure 3 shows that there are significant differences between hippocampal and cortical networks. However, given that the distributions of values can overlap and yet still be statistically significant (e.g. firing rate at $21 \mathrm{DIV}$; Figure 3A), we cannot use individual features to reliably discriminate between the two types of network. We therefore used machine-learning techniques to address two related questions:

1. Out of the 11 features, which are the most important for discriminating hippocampal versus cortical networks?

2. Given a recording of a network at a given age, is it possible to predict the identity (hippocampal or cortical) of the network?

We therefore translated each 15-minute recording into an 11-dimensional vector, with element $i$ of the vector storing the value of feature $i$ measured from the recording. This vector is described below as a feature vector of the recording. 


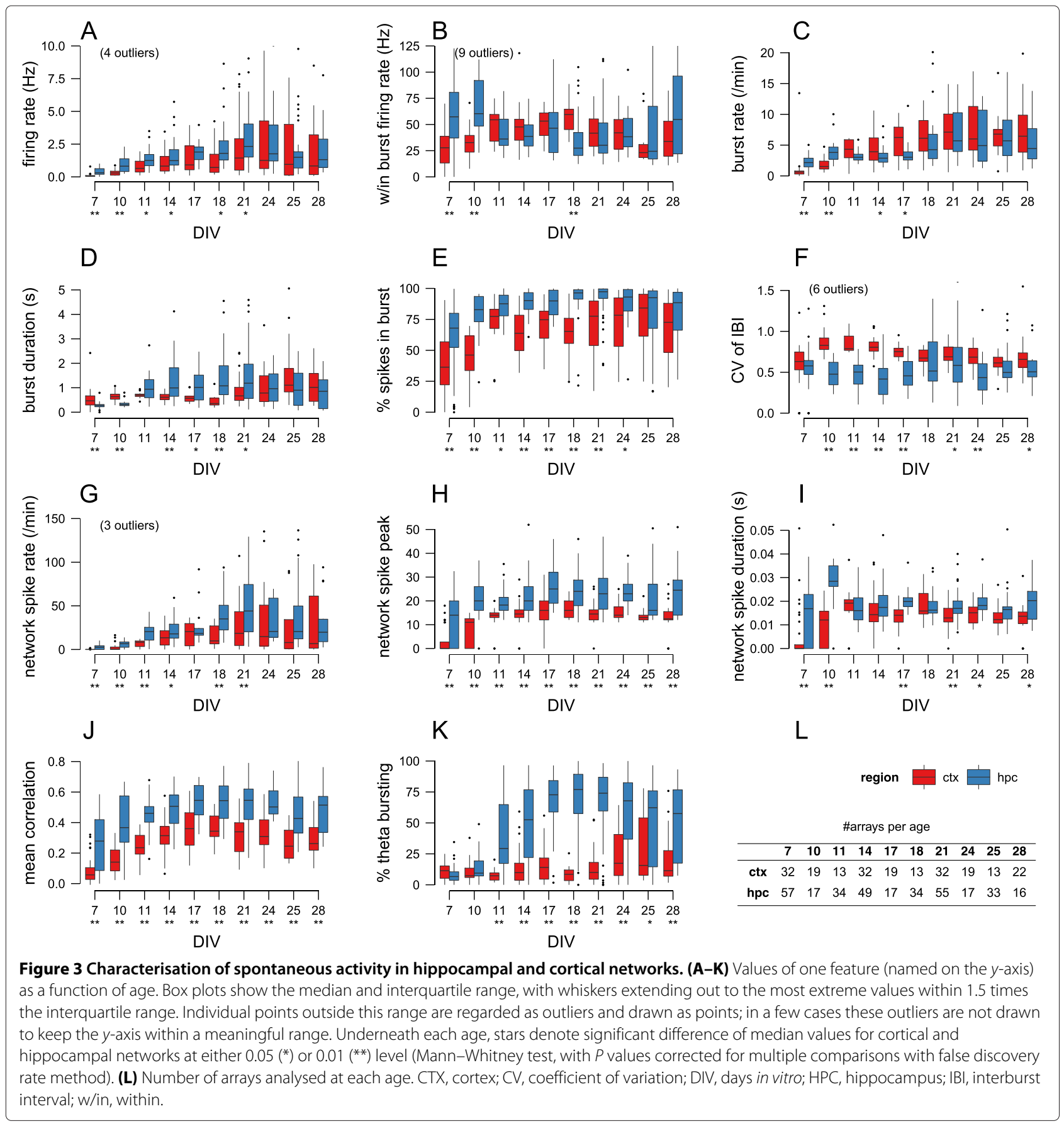

\section{Principal component analysis}

If there is a consistent difference in the properties of hippocampal and cortical recordings, we would hope that the corresponding feature vectors cluster into two distinct regions. However, as these feature vectors are 11dimensional, we must first reduce their dimensionality to visualise them. Many such dimensionality-reduction techniques are available; we chose to use the best-known method, principal component analysis. Figure 4 shows the projection of the feature vectors at three different ages down into two-dimensional space. At $7 \mathrm{DIV}$, there is significant overlap between the hippocampal and cortical recordings, which might suggest that it is hard to discriminate between the two types of recordings; however, at 14 and $21 \mathrm{DIV}$, the recordings from the same cell type cluster and there is significant separation of the hippocampal and cortical recordings. The graphs underneath each scatter plot show the cumulative percentage of 

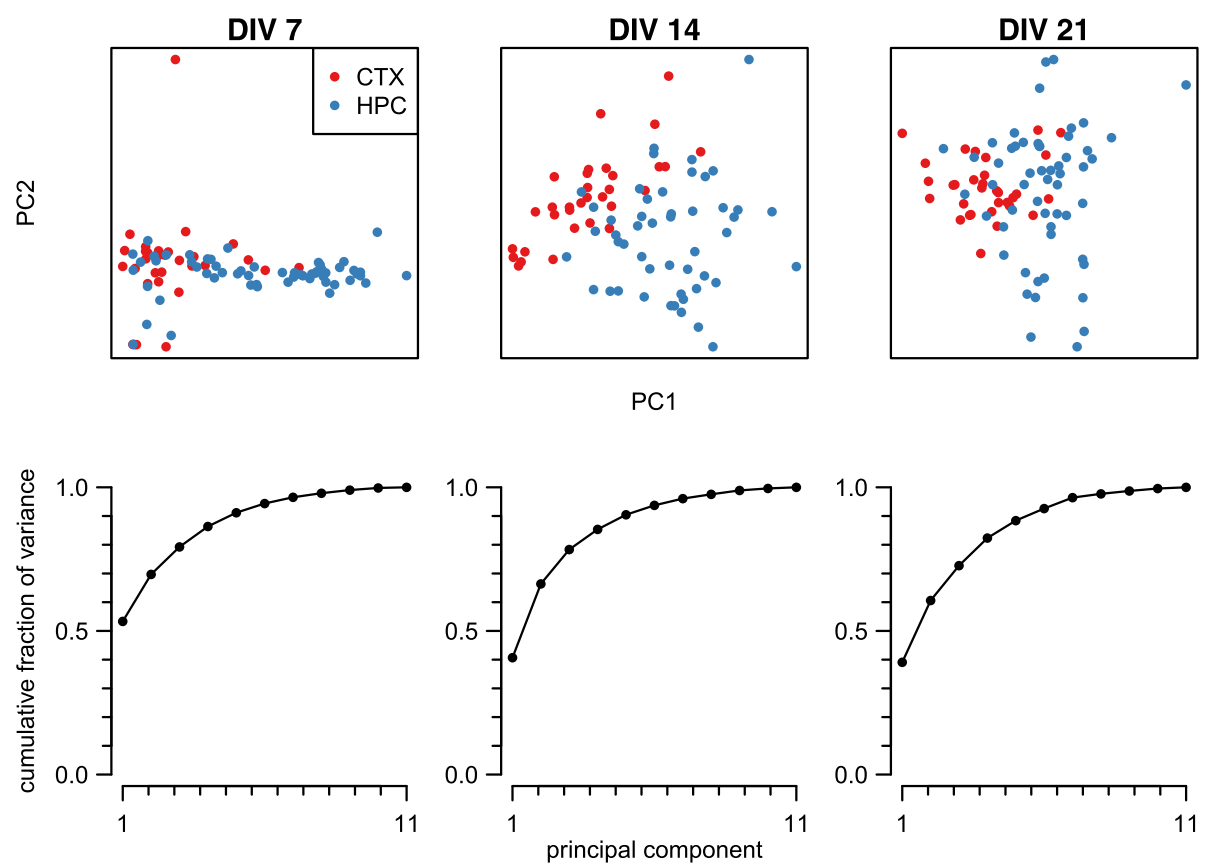

Figure 4 Principal component analysis of hippocampal and cortical feature vectors. Each column represents a principal component analysis of the 11-dimensional feature vectors of all recordings at a given age (days in vitro). In the scatter plot, each point represents one recording projected down into the two dimensions that account for maximal variance and is coloured according to its cell type. Each graph shows the cumulative fraction of variance accounted for by the principal components. CTX, cortex; DIV, days in vitro; HPC, hippocampus; PC, principal component.

variance accounted for by the components. In each case, the first two principal components account for at least $60 \%$ of the variance.

\section{Classification of recordings}

The principal component analysis suggests that, especially at the later ages, the feature vectors contain sufficient signal to discriminate between hippocampal and cortical networks. However, given the overlap between clusters, we next used classification techniques to quantify the degree to which the two classes of recording can be separated. We used two classification methods, detailed below. In both methods, two-thirds of the feature vectors at a given age are used to train a classifier to discriminate between the two types of recording. The remaining onethird of the feature vectors are then used as a test set to evaluate how well the classifier performs on data unseen during training.

We first used classification trees [18]. We built ten classifiers, one per age studied, to test whether the recordings could be grouped into cortical or hippocampal networks. We found that for any given age, the prediction accuracy of the trees was high - usually over 75\% correct, depending on the age of the recording. (Performance would be $50 \%$ if there were no information to distinguish the two types of recording.) Once these trees had been built, we were able to interrogate them to find out which features were dominant in driving the classification of the network. At different ages, unsurprisingly, different features were dominant, but an overall trend clearly emerged when we averaged across developmental ages. Table 1 lists the features in decreasing order of their importance, along with their relative score (column 2).

Out of the 11 features, three stood out. The most important was $\mathrm{CV}$ of IBI; this is a measure of firing regularity, which tends to be higher in hippocampal networks. Second, theta bursting is a key indicator (once it emerges at DIV 11) of hippocampal networks. Third, mean correlation is one of the top three features roughly half the time, and again is higher in hippocampal networks.

We chose classification trees as our first classification method primarily because of their simplicity (there are no free parameters) and ability to assess easily which features are driving the classification. Although their classification performance was good, we compared their performance against another common machine-learning technique, namely support vector machines (SVMs). We found that the SVM classifiers tended to result in slightly higher classification accuracy than the classification trees; e.g. when all 11 features were used, performance was $75 \%$ to $97 \%$ across ages (bottom row of Table 1).

Finally, given that our classifier trees provide us with a natural ordering of the importance of features, we asked 
Table 1 Classifier performance at discriminating cortical from hippocampal cultures

\begin{tabular}{llllllllllll}
\hline & & \multicolumn{10}{c}{ Percentage correct at given age (DIV) } \\
\cline { 3 - 10 } Feature & Score & $\mathbf{7}$ & $\mathbf{1 0}$ & $\mathbf{1 1}$ & $\mathbf{1 4}$ & $\mathbf{1 7}$ & $\mathbf{1 8}$ & $\mathbf{2 1}$ & $\mathbf{2 4}$ & $\mathbf{2 5}$ & $\mathbf{2 8}$ \\
\hline CV of IBI & 1.00 & 64 & 85 & 88 & 89 & 84 & 71 & 61 & 72 & 73 & 60 \\
Theta burst & 0.84 & 64 & 82 & 96 & 88 & 91 & 89 & 84 & 86 & 74 & 63 \\
Mean correlation & 0.50 & 67 & 89 & 96 & 91 & 86 & 94 & 89 & 84 & 75 & 75 \\
Burst duration & 0.33 & 72 & 86 & 97 & 91 & 92 & 90 & 89 & 82 & 77 & 83 \\
Burst rate & 0.32 & 78 & 84 & 97 & 92 & 90 & 95 & 89 & 91 & 74 & 78 \\
\% of spikes in bursts & 0.25 & 82 & 86 & 92 & 94 & 92 & 94 & 89 & 87 & 77 & 79 \\
Firing rate & 0.21 & 83 & 84 & 94 & 94 & 90 & 94 & 92 & 89 & 74 & 78 \\
NS peak & 0.19 & 83 & 86 & 91 & 95 & 91 & 96 & 91 & 88 & 76 & 79 \\
NS duration & 0.19 & 83 & 89 & 90 & 92 & 89 & 93 & 93 & 86 & 72 & 78 \\
Within-burst firing rate & 0.16 & 83 & 89 & 96 & 93 & 92 & 96 & 93 & 87 & 77 & 79 \\
NS rate & 0.11 & 83 & 88 & 92 & 92 & 90 & 97 & 93 & 85 & 76 & 75 \\
\hline
\end{tabular}

Features are listed in decreasing order of importance (score; column 2) normalised to the top score. The following numbers in each row $i=1, \ldots, 11$ are the mean percentage of correct classifications at each age using the top $i$ features. CV, coefficient of variation; DIV, days in vitro; IBI, interburst interval; NS, network spike.

how performance varied as we reduced the number of features that each recording is represented by. We found that performance remained high even as the number of features was gradually reduced (moving up through the rows of Table 1). It is clear, however, that multiple features are required for good classification; when only the single most important feature is used (top row of Table 1), performance was only just above chance at some ages. However, with only three or four features, we obtained good performance across all ages.

In conclusion, the results from the classifiers tell us that three features of network activity (CV of IBI, theta bursting and mean correlation) are strong predictors of whether a recording is from a hippocampal or a cortical network.

\section{Discussion}

We have found that cultured networks of either hippocampal or cortical neurons generate spontaneous activity. These patterns of activity change during development and already by 7 DIV significant differences in their activity patterns begin to emerge. We have developed a quantitative framework for examining these activity patterns. By calculating 11 features of activity patterns, we can represent each recording of spontaneous activity as a point in (11-dimensional) feature space. When we examine recordings from any one given developmental age, we find that recordings from the same neuronal type (cortical or hippocampal) cluster in this feature space such that we can reliably discriminate between hippocampal and cortical networks.

Furthermore, out of the 11 features, we find that three are critically important for this classification in feature space. First, the CV of IBI was most important on average in driving the classification. Hippocampal spike trains tend to fire in bursts that are more regularly spaced than spike trains from cortical neurons. Second, after about 11 DIV, most electrodes in hippocampal recordings detect theta bursting, compared to a minority in cortical recordings. Third, the mean correlation between pairs of electrodes tends to be higher in hippocampal networks. These three measures are all relatively simple, and measure activity on either a single-electrode level or from pairs of electrodes. By contrast, although significant differences were found in the network spike measures, most importantly, the peak of network spikes (Figure 3F), these measures were not deemed to be critical in classification.

We have deliberately chosen simple features to characterise spiking activity to see if they suffice to discriminate between cortical and hippocampal networks. It is entirely likely that other more complicated measures of activity, particularly at the network level, may also reveal clear differences between these two types of network [10]. For example, network connectivity measures have been used to explore differences in spontaneous activity patterns between mature hippocampal and cortical networks in a range of frequency bands [19]. However, the simple measures we have chosen here suffice to differentiate the two types of network reliably. Likewise, even higher classification performance may be possible with more complex machine-learning techniques. However, our primary interest was to see whether in principle the feature space can be reliably separated with standard approaches [18]. Similar machine-learning methods are not yet routinely used in analysing spontaneous activity, although see [20] for a recent example showing how single-cell activity could be classified as either in vivo or in vitro. Finally, 
with the advent of a new generation of higher density MEAs containing up to 4,096 electrodes [21], it is likely that there are much richer patterns of activity than we describe here.

We believe that our framework lends itself nicely to many applications, for example in neurotoxicity testing where spontaneous activity from a network is recorded whilst it is exposed to a particular compound [7]. By building up a representative feature space of recordings from compounds known to be either toxic or safe, our approach can be used to predict the toxicity of novel compounds. This idea builds upon earlier work where mean profiles of activity in each condition were used as simple classifiers [22]. More recently, SVMs were used for toxicity prediction [23]. We imagine that our approach could also be used to detect the impact of particular genetic mutations, given earlier work suggesting that there may be significant differences in network activity [24].

\section{Limitations and future work}

Our current work describes key quantitative differences in cortical and hippocampal spontaneous activity, but as yet we cannot explain what mechanisms might underlie these differences. One simple explanation might be that the two networks develop at different rates, and so comparing networks at the same age (measured as days in vitro) might be inappropriate. We also do not yet know whether the two types of network have different cellular compositions, such as the fraction of inhibitory interneurons or glia; even different fractions of $\mathrm{GABA}_{\mathrm{A}}$ and $\mathrm{GABA}_{\mathrm{B}}$ mediated inhibition can significantly regulate network states [25]. Alternatively, these differences in activity patterns could be driven by molecular differences in individual neurons and synapses, or by differences in network connectivity [10].

One potential concern about our current findings is that neuronal activity in vitro may provide little insight about neuronal activity in vivo. It is possible that other in vitro methods, such as brain slices, might produce more realistic activity patterns of spontaneous activity [26]. Recent machine-learning approaches have suggested, however, that cultured neurons generate spontaneous activity patterns more similar to in vivo activity than activity from organotypic slices [20]. However, we make no strong claims about whether our recordings from networks of cultured neurons can tell us about in vivo activity patterns; we believe that both cultured networks and slice preparations are only simple approximations to in vivo networks. Furthermore we believe the utility of in vitro approaches is that it we can readily study network properties under different environmental, genetic or pharmacological conditions.

\section{Conclusions}

We report key differences in the developmental spontaneous activity patterns of cultured networks of hippocampal and cortical neurons. We have proposed a quantitative framework for evaluating these patterns. Our database of recordings and computer programs are all freely available for others to build upon. Future work in this area could be to dissect the cellular or network mechanisms driving the differences in network activity. For example, the differences between the cortical and hippocampus cultures could reflect molecular differences in cells or synapse or cellular differences in the populations of cells. Alternatively, differences in functional connectivity might partially account for these results [10]. Dissecting these differences will require a detailed understanding of the diversity of cell types defined by singlecell transcriptomes in these brain regions, which is still lacking.

\section{Methods}

\section{Primary neuronal culture}

Primary cultures of dissociated hippocampal and cortical neurons were prepared from mice at embryonic day (E) 17 to 18. Hippocampi and cortices were dissected from E17.5 mouse embryos (two to four, pooled) and transferred to papain (10 units/mL, Worthington, Lakewood, NJ, USA) for $22 \mathrm{~min}$ at $37^{\circ} \mathrm{C}$. Cells were manually dispersed in Dulbecco's modified Eagle's medium containing $10 \% \mathrm{v} / \mathrm{v}$ foetal bovine serum and centrifuged twice at $400 \mathrm{~g}$ for $3.5 \mathrm{~min}$. The final pellet was resuspended in Neurobasal/B27 supplemented with $0.5 \mathrm{mM}$ Gln (Invitrogen, Paisley, UK), and dissociated cells $\left(2 \times 10^{5}\right.$ per dish $)$ were seeded in the centre of poly-D-lysine/laminincoated MEAs (60MEA200/30-Ti, Multi Channel Systems, Reutlingen, Germany) containing $600 \mu$ full Neurobasal medium. Zero-evaporation lids [27] were fitted and the MEAs housed in tissue culture incubators maintained humidified at $37^{\circ} \mathrm{C}$ and $5 \% \mathrm{CO}_{2} / 95 \%$ air. Twenty-four hours post-plating, sample MEAs were placed on an inverted microscope with a heated stage (Axiovert 200; Zeiss, Cambridge, UK) and photographed through a $32 \times$ phase objective at five different fields of view. These images were then analysed with CellProfiler [28] to quantify the neuronal density over the electrode array, giving an average value of 1,500 cells $/ \mathrm{mm}^{2}$.

At 3 to 4 days in vitro, cultures were fed by replacing $200 \mu \mathrm{l}$ medium with pre-warmed fresh full Neurobasal medium. Cultures were subsequently fed using the same method after each recording, equating to a one-third medium change twice per week.

All procedures were performed in accordance with the United Kingdom Animals (Scientific Procedures) Act 1986. The mouse line used in this study was C57BL/6Tyr ${ }^{\text {c-Brd }}$ (C57; albino C57BL/6). 


\section{Multielectrode array recording}

MEAs and all data acquisition hardware and software were from MultiChannel Systems (Reutlingen, Germany). Pairs of MEAs were interfaced with duplex 60 channel amplifiers and 15-minute recordings of spontaneous action potentials were made twice per week during the 4 weeks following plating. MEAs were heated and kept under a light flow of $5 \% \mathrm{CO}_{2} / 95 \%$ air during recordings. Signals were digitised with a 128-channel analogue/digital converter card at a rate of $25 \mathrm{kHz}$ and filtered $(100 \mathrm{~Hz}$ high pass) to remove low-frequency events and baseline fluctuations. Action potentials were detected by the crossing of a threshold set to a fixed level of $-20 \mu \mathrm{V}$, which typically approximated to 6 to 8 standard deviations from the baseline noise level. Record samples $(1 \mathrm{~ms}$ pre- and $2 \mathrm{~ms}$ post-crossing of threshold) confirmed the characteristic action potential waveform. Application of tetrodotoxin $(1 \mu \mathrm{M})$ totally abolished spiking activity, confirming the absence of false positive event detection using these methods. Spikes were not sorted to distinguish signals generated by individual neurons, so represent multiunit activity. Action potential timestamps were extracted using batch scripts written for NeuroExplorer [29] and analysed using software developed in the $\mathrm{R}$ statistical programming environment to compute parameters that quantitatively describe network activity. In total, 214 recordings were taken from 32 arrays of cultured cortical neurons, and 329 recordings from 61 arrays of cultured hippocampal neurons.

\section{Data analysis}

To summarise a 15-minute recording of network activity, we computed the following features. As all recordings detected activity from multiple electrodes, we calculated summary scalar values (termed the array value below) by summarising the information from multiple electrodes. In this way, each recording was then represented as an 11-dimensional vector.

1. Firing rate The mean firing rate of each electrode was calculated. The array value was the median of all electrode firing rates.

2. Within-burst firing rate Bursts were detected independently on each electrode using our implementation of the max interval method from Neuroexplorer [29]. The parameters for burst detection are given in Table 2. For each electrode we calculated the mean of the firing rate during each burst. The array value was the median of the within-burst firing rates, ignoring electrodes where no bursts were detected.

3. Burst rate For each electrode we calculated the number of bursts per minute. The array value was as per feature 2.
Table 2 Burst detection parameters

\begin{tabular}{ll}
\hline Parameter & Value \\
\hline Maximum beginning interspike interval & $0.1 \mathrm{~s}$ \\
Maximum end interspike interval & $0.25 \mathrm{~s}$ \\
Minimum interburst interval & $0.8 \mathrm{~s}$ \\
Minimum burst duration & $0.05 \mathrm{~s}$ \\
Minimum number of spikes in a burst & 6 \\
\hline
\end{tabular}

4. Burst duration The electrode value was the mean duration of bursts on that electrode. The array value was as per feature 2 .

5. Fraction of spikes in bursts The electrode value was the total number of spikes classified as belonging to a burst divided by the total number of spikes on the electrode. The array value was as per feature 2 .

6. CV of IBI The electrode value was the CV (standard deviation divided by mean) of the IBIs. The array value was as per feature 2 .

7. Rate of network spikes Network spikes were defined as the array-wide average population activity [13]. It is defined by dividing time into small bins (here $3 \mathrm{~ms}$ ) and counting the number of electrodes that generated at least one action potential in that bin. A network spike is then defined as the period when more than a threshold number of electrodes (here $n=10$ ) are simultaneously active. The array value was the number of network spikes per minute.

8. Network spike peak During each network spike we found the maximum number of active electrodes. The array value was the median of the values from each network spike in a recording.

9. Network spike duration The duration of each network spike was the time (in seconds) that the count of active electrodes exceeded the threshold value. The array value was as per feature 8 .

10. Mean correlation Given two different spike trains from the recording, we calculated the correlation between them using the spike time tiling coefficient [14] with the coincidence window of $\Delta t=5 \mathrm{~ms}$. (We also tried $\Delta t=50 \mathrm{~ms}$ and $0.5 \mathrm{~ms}$, but results were qualitatively similar.) The array value was the mean of all distinct pairs of electrodes.

11. Fraction of electrodes exhibiting theta bursting For each electrode, the log ISI histogram was calculated and smoothed with the default kernel density estimation routine in $\mathrm{R}$. A spike train was classed as showing theta bursting if a peak was present in the 4 to $10 \mathrm{~Hz}$ band of the histogram. The array value was the fraction of electrodes on the array that were classified as theta bursting. 
The Mann-Whitney test was used to test whether the median array values at any given developmental age differed between the hippocampal and cortical networks. The $P$ values were then corrected for multiple comparisons using the false discovery rate method [30].

\section{Clustering and classification}

Standard principal component analysis was performed (with variance normalisation for each feature) for all feature vectors of any given age. Two standard machinelearning classifiers were tested: classification trees with boosting (random forests) and SVMs using radial kernel functions with $\gamma=1 / 11$ [18]. For each age, we built binary classifiers to predict the region (CTX/HPC) based upon the 11 features measured from each recording. For both classifiers, we used two-thirds of the recordings as training data, with the remaining one-third used as test data. Performance is reported as mean percentage of correct classifications, averaged over 500 repeats using different splits of the data into training and test sets. The classification tree approach allows us to assess the relative importance of features by measuring the degree to which they decrease the Gini index ([18], p. 319). These values were normalised to the value of the top-performing feature.

\section{Data and code availability}

Statistical analysis was performed in the R programming environment using the SJEMEA package [31]. Data files containing the spike times from all recordings analysed here were stored in the HDF5 format using the framework created for spontaneous activity in retinal recordings [32]. The only addition to the framework was a new metadata item / meta/region containing either the phrase 'CTX' or 'HPC' depending on the network type. All data files and analysis code relating to this paper are freely available [33]. This includes all the material required to regenerate the figures and table in this article.

\begin{abstract}
Abbreviations
CTX: Cortex; CV: Coefficient of variation; DIV: Days in vitro; HPC: Hippocampus; IBI: Interburst interval; ISI: Interspike interval; MEA: Multielectrode array; NS: Network spike; SVM: Support vector machine.
\end{abstract}

\section{Competing interests}

The authors declare that they have no competing interests.

\section{Authors' contributions}

PC, AM and SGNG conceived and designed the project. PC and AM performed all the experiments. EC and SJE provided analysis tools. PC, EC and SJE analysed the data. SJE drafted the manuscript. PC, EC, SGNG and SJE edited the manuscript. All authors read and approved the final version of the manuscript.

\section{Acknowledgements}

Thanks to Diana Hall, Johannes Hjorth and Ole Paulsen for comments on this work. PC and AM were supported by the Wellcome Trust Genes to Cognition programme. PC received additional support from the Biotechnology and Biological Sciences Research Council (BB/H008608/1). EC was supported by a Wellcome Trust PhD studentship and Cambridge Biomedical Research Centre studentship. SJE was supported by an Engineering and Physical Sciences Research Council grant (EP/E002331/1).

\section{Author details}

${ }^{1}$ Genes to Cognition Programme, Wellcome Trust Sanger Institute, Genome Campus, Hinxton, CB10 1SA, UK. ${ }^{2}$ Current address: Department of Physiology, Development and Neuroscience, Physiological Laboratory, Downing Street, Cambridge, CB2 3EG, UK. ${ }^{3}$ Cambridge Computational Biology Institute, University of Cambridge, Wilberforce Road, Cambridge, CB3 OWA, UK. ${ }^{4}$ Current address: Centre for Integrative Physiology, University of Edinburgh School of Biomedical Sciences, Edinburgh, EH8 9XD, UK. ${ }^{5}$ Centre for Clinical Brain Sciences and Centre for Neuroregeneration, Chancellors Building, Edinburgh University, 49 Little France Crescent, Edinburgh, EH16 4SB, UK.

Received: 1 October 2014 Accepted: 11 December 2014

Published online: 28 January 2015

\section{References}

1. Blankenship AG, Feller MB. Mechanisms underlying spontaneous patterned activity in developing neural circuits. Nat Rev Neurosci. 2010;11:18-29.

2. Leinekugel X, Khazipov R, Cannon R, Hirase H, Ben-Ari Y, Buzsáki G. Correlated bursts of activity in the neonatal hippocampus in vivo. Science. 2002;296:2049-52.

3. Khazipov R, Sirota A, Leinekugel X, Holmes GL, Ben-Ari Y, Buzsáki G. Early motor activity drives spindle bursts in the developing somatosensory cortex. Nature. 2004;432:758-61.

4. Hanganu IL, Ben-Ari Y, Khazipov R. Retinal waves trigger spindle bursts in the neonatal rat visual cortex. J Neurosci. 2006:26:6728-36.

5. Seelke AMH, Blumberg MS. Developmental appearance and disappearance of cortical events and oscillations in infant rats. Brain Res. 2010;1324:34-42.

6. Colonnese MT, Khazipov R. Slow activity transients' in infant rat visual cortex: a spreading synchronous oscillation patterned by retinal waves. J Neurosci. 2010;30:4325-37.

7. Johnstone AFM, Gross GW, Weiss DG, Schroeder OHU, Gramowski A, Shafer TJ. Microelectrode arrays: a physiologically based neurotoxicity testing platform for the 21st century. Neurotoxicology. 2010;31:331-50.

8. Wagenaar DA, Pine J, Potter SM. An extremely rich repertoire of bursting patterns during the development of cortical cultures. BMC Neurosci. 2006;7:11

9. Rolston JD, Wagenaar DA, Potter SM. Precisely timed spatiotemporal patterns of neural activity in dissociated cortical cultures. Neuroscience. 2007;148:294-303.

10. Soriano J, Rodríguez Martínez M, Tlusty T, Moses E. Development of input connections in neural cultures. Proc Natl Acad Sci USA. 2008;105: 13758-63.

11. Gullo F, Manfredi I, Lecchi M, Casari G, Wanke E, Becchetti A. Multi-electrode array study of neuronal cultures expressing nicotinic $\beta 2$-V287L subunits, linked to autosomal dominant nocturnal frontal lobe epilepsy. An in vitro model of spontaneous epilepsy. Front Neural Circuits. 2014;8:87.

12. Lisman JE. Bursts as a unit of neural information: making unreliable synapses reliable. Trends Neurosci. 1997;20:38-43.

13. Eytan D, Marom S. Dynamics and effective topology underlying synchronization in networks of cortical neurons. J Neurosci. 2006;26: 8465-76.

14. Cutts CS, Eglen SJ. Detecting pairwise correlations in spike trains: an objective comparison of methods and application to the study of retinal waves. J Neurosci. 2014;34:14288-303.

15. Wong RO, Meister M, Shatz CJ. Transient period of correlated bursting activity during development of the mammalian retina. Neuron. 1993;11: 923-38.

16. Godfrey KB, Eglen SJ. Theoretical models of spontaneous activity generation and propagation in the developing retina. Mol Biosyst. 2009;5: 1527-35.

17. Buzsáki G. Theta oscillations in the hippocampus. Neuron. 2002;33:325-40.

18. James G, Witten D, Hastie T, Tibshirani R. An Introduction to Statistical Learning: With Applications in R. New York: Springer; 2014.

19. Ito $S$, Yeh FC, Hiolski E, Rydygier $P$, Gunning DE, Hottowy $P$, et al. Large-scale, high-resolution multielectrode-array recording depicts 
functional network differences of cortical and hippocampal cultures. PLoS One. 2014;9:e105324.

20. Okamoto K, Ishikawa T, Abe R, Ishikawa D, Kobayashi C, Mizunuma M, et al. Ex vivo cultured neuronal networks emit in vivo-like spontaneous activity. J Physiol Sci. 2014;64:421-31.

21. Maccione A, Hennig MH, Gandolfo M, Muthmann O, van Coppenhagen J, Eglen SJ, et al. Following the ontogeny of retinal waves pan-retinal recordings of population dynamics in the neonatal mouse. J Physiol. 2013;592:1545-63.

22. Gramowski A, Jügelt K, Weiss DG, Gross GW. Substance identification by quantitative characterization of oscillatory activity in murine spinal cord networks on microelectrode arrays. Eur J Neurosci. 2004;19:2815-25

23. Mack CM, Lin BJ, Turner JD, Johnstone AFM, Burgoon LD, Shafer TJ. Burst and principal components analyses of MEA data for 16 chemicals describe at least three effects classes. Neurotoxicology. 2014;40:75-85.

24. MacLaren EJ, Charlesworth P, Coba MP, Grant SGN. Knockdown of mental disorder susceptibility genes disrupts neuronal network physiology in vitro. Mol Cell Neurosci. 2011;47:93-9.

25. Mann EO, Kohl MM, Paulsen O. Distinct roles of GABA and GABAB receptors in balancing and terminating persistent cortical activity. J Neurosci. 2009;29:7513-18.

26. Mao BQ, Hamzei-Sichani F, Aronov D, Froemke RC, Yuste R. Dynamics of spontaneous activity in neocortical slices. Neuron. 2001;32:883-98.

27. Potter SM, DeMarse TB. A new approach to neural cell culture for long-term studies. J Neurosci Methods. 2001;110:17-24.

28. Carpenter AE, Jones TR, Lamprecht MR, Clarke C, Kang IH, Friman O, et al. CellProfiler: image analysis software for identifying and quantifying cell phenotypes. Genome Biol. 2006;7:R100

29. Nex Technologies. NeuroExplorer Manual. 2012. [http://www. neuroexplorer.com/downloads/NeuroExplorerManual.pdf]

30. Benjamini Y, Hochberg Y. Controlling the false discovery rate: a practica and powerful approach to multiple testing. J R Stat Soc Series B Stat Methodol. 1995;57:289-300.

31. R Core Team. R: A Language and Environment for Statistical Computing Vienna, Austria; 2014. [http://www.r-project.org]

32. Eglen SJ, Weeks M, Jessop M, Simonotto J, Jackson T, Sernagor E. A data repository and analysis framework for spontaneous neural activity recordings in developing retina. Gigascience. 2014;3:3.

33. Genes to Cognition: Hippocampus vs Cortex project. [http://github.com/ sje30/g2chvc]

\section{Submit your next manuscript to BioMed Central} and take full advantage of:

- Convenient online submission

- Thorough peer review

- No space constraints or color figure charges

- Immediate publication on acceptance

- Inclusion in PubMed, CAS, Scopus and Google Scholar

- Research which is freely available for redistribution

Submit your manuscript at www.biomedcentral.com/submit
C Biomed Central 